This item was submitted to Loughborough's Research Repository by the author.

Items in Figshare are protected by copyright, with all rights reserved, unless otherwise indicated.

\title{
The vibrations of radial ball bearings
}

PLEASE CITE THE PUBLISHED VERSION

PUBLISHER

Professional Engineering Publishing / @ IMECHE

VERSION

VoR (Version of Record)

LICENCE

CC BY-NC-ND 4.0

REPOSITORY RECORD

Rahnejat, Homer, and Ramsey Gohar. 2019. "The Vibrations of Radial Ball Bearings". figshare. https://hdl.handle.net/2134/4812. 
This item was submitted to Loughborough's Institutional Repository (https://dspace.lboro.ac.uk/) by the author and is made available under the following Creative Commons Licence conditions.

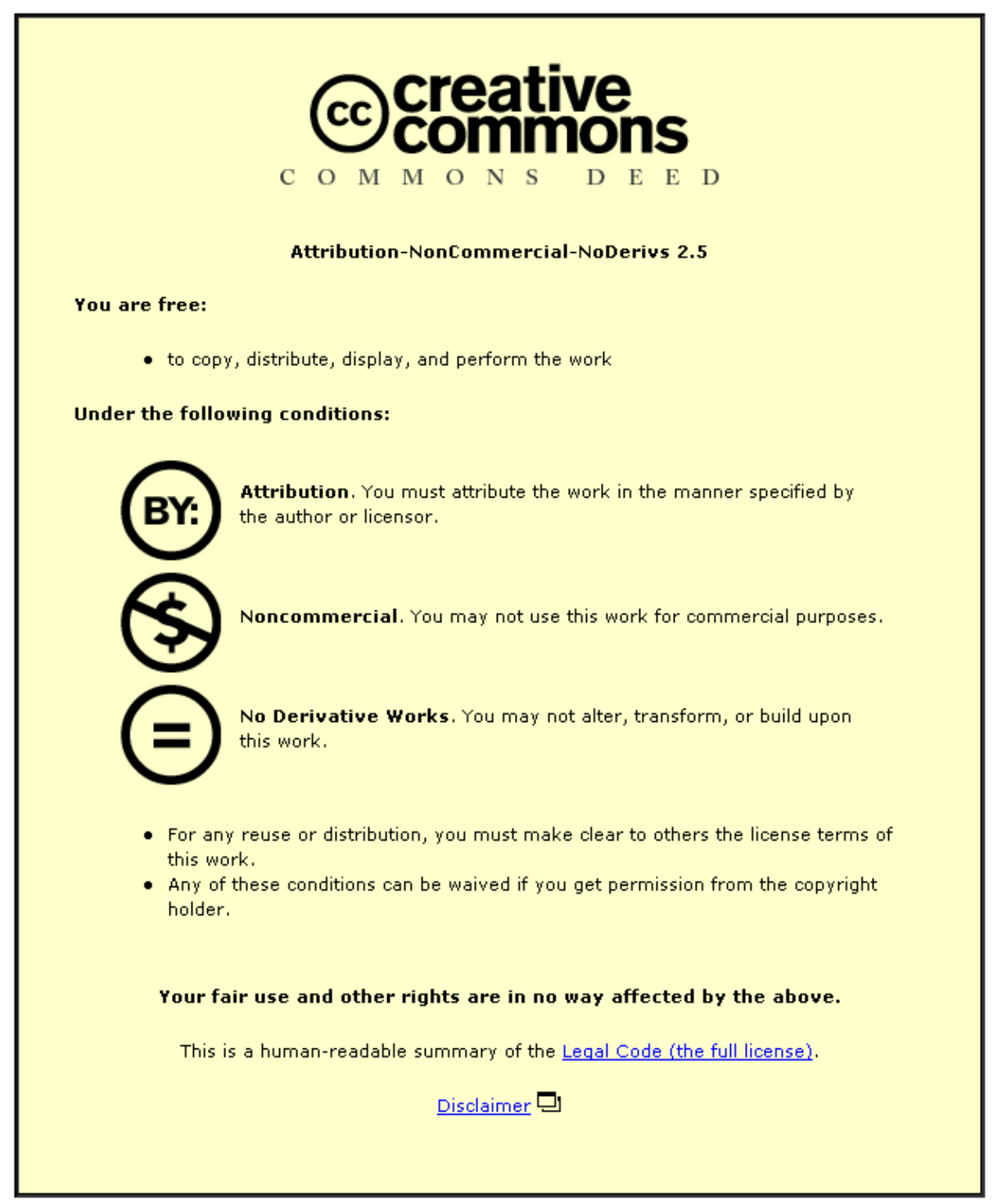

For the full text of this licence, please go to: http://creativecommons.org/licenses/by-nc-nd/2.5/ 


\section{The vibrations of radial ball bearings}

H Rahnejat, BSc, MSc and R Gohar, BSc, DIC, PhD, CEng, MIMechE

Department of Mechanical Engineering, Imperial College of Science and Technology, Exhibition Road, London SW7

This paper presents a theoretical analysis of the vibration response of a rotating rigid shaft supported by two radial deep-groove lubricated ball bearings. The bearings and their oil films are approximated to a set of non-linear elastic springs and dampers rotating relative to the shaft when it is subjected to a rotating unbalance or inner race surface waviness. Under gravity load, the shaft's initial vibration is damped down to a limit cycle operating at a quasi-simple harmonic frequency of small amplitude. Rotating unbalance and surface features introduce further significant frequencies which influence the output response.

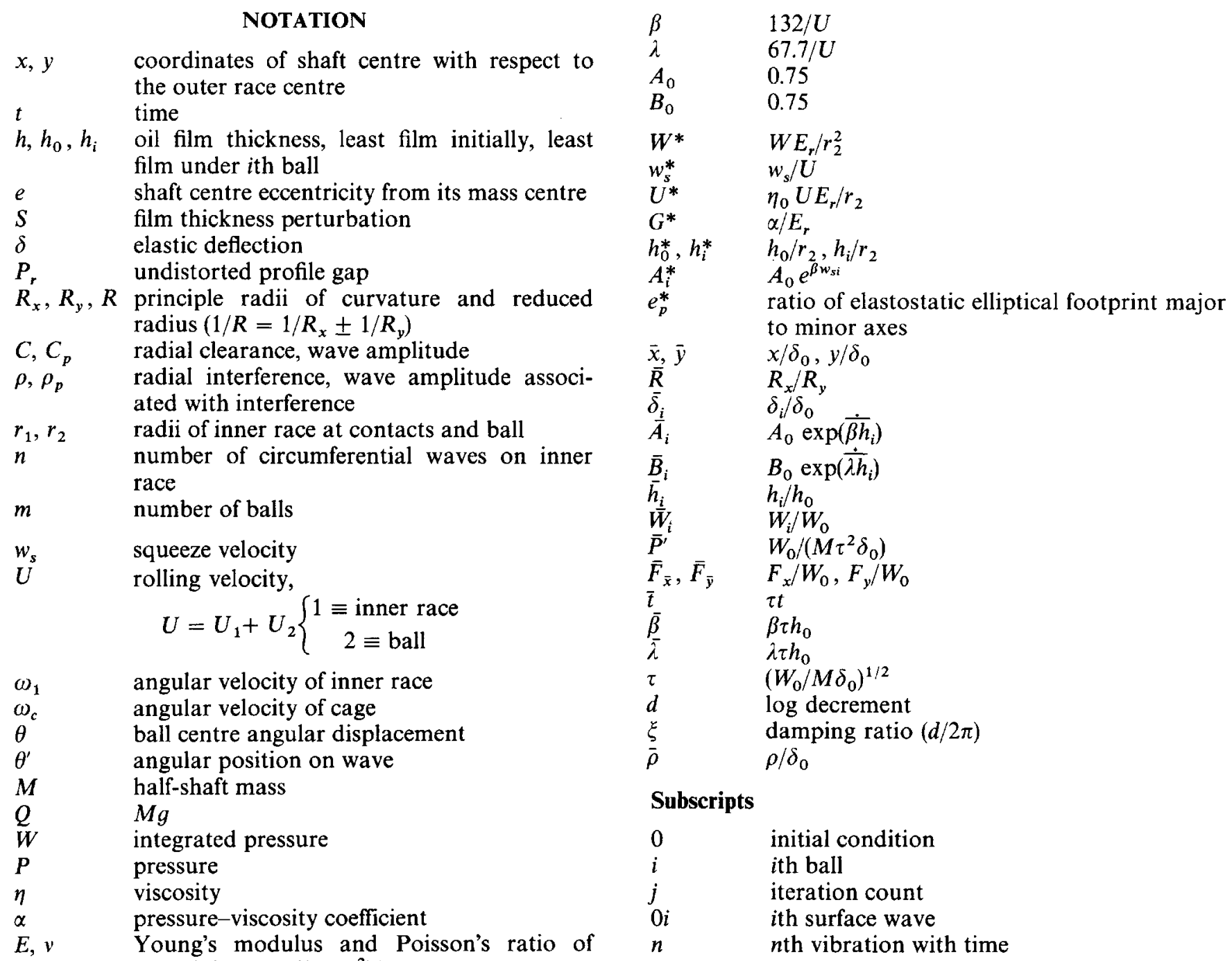

\section{INTRODUCTION}

The stiffness, rotational accuracy and vibration characteristics of a high-speed spindle are partly controlled by the rolling element bearings that support it. These bearings, together with the oil films, can be considered as non-linear springs and squeeze film dampers rotating round the spindle. An analysis of the bearings' dynamic behaviour is therefore important if a prediction of the system vibration response is to be made. It has also long been recognized that the manufacturing quality

The MS was received on 11 June 1984 and was accepted for publication on January 1985 .

$13.50 \mathrm{GN} / \mathrm{m}^{3 / 2}$ 
and care of assembly of the bearings influences their noise levels as well as their life expectancy (1).

Some recent theoretical and experimental studies have examined the stiffness and damping characteristics of disc machines (2) and angular contact bearings (3), the conclusion being that the oil films themselves can act as squeeze film dampers, while, for the bearings, some dry friction damping may also occur between the outer race and its housing. Other experiments on machine tool spindles have explained the influence of preload on dynamic stiffness and vibration characteristic of angular contact bearings $(4,5)$.

In the work reported below, a theoretical study is made of the vibration response of a rigid horizontal shaft having a mid-span load and supported by two deep-groove radial ball bearings, thus allowing for twodimensional motion of its centre in the vertical plane. Most of the models employed assume the bearings to be geometrically perfect but possessing various amounts of radial interference. These bearings are then allowed to vibrate freely or be subjected to rotating unbalance. Imperfect models assume some form of prescribed race surface waviness, and again system response is found. In all the examples quoted the rolling elements are considered massless and are fully lubricated during their oribital paths.

\section{GOVERNING EQUATIONS}

\subsection{Oil film thickness}

Hitherto, published expressions for the oil film thickness under elastohydrodynamic lubrication (e.h.l.) conditions have not considered the surface squeeze velocity (normal to the surfaces) when combined with the rolling velocity. When a rotating ball bearing is subjected additionally to a periodic or step load, squeeze forces will be encountered as well as those caused by the normal rolling motion. These time-dependent squeeze forces must be included in any analysis. There are several solutions to the e.h.l. problem when considering squeeze motion only $(6,7)$. The usual method is to use a marching procedure, starting with the moving member far out as an undistorted elastic body and observe its distortion as its uniform geometric centre velocity drives it towards the other rigid stationary surface through the oil film. Points on the moving oil film roof at any time do not generally have equal velocities during the squeezing motion. Herrebrugh (6), for example, studied the squeeze action of an elastic cylinder approaching a plane through an oil film and assumed a uniform surface velocity in the region of pressure. The different film shapes he obtained at different times were a measure of the inaccuracy of the uniform velocity assumption, but were considered as a first approximation to a complete solution. One general conclusion reached was that, except for very thin films, a rigid body assumption is valid. However, as the situation in vibrating bearings can involve thin films, the rigid body assumption cannot be made in obtaining a regression formula for the relation between material properties, film thickness, load, viscosity, rolling speed and surface squeeze speed, for any rolling element. When the dynamics of the problem is considered, the surface squeeze speed, although considered uniform, is different from the velocity of a remote point on the element. A

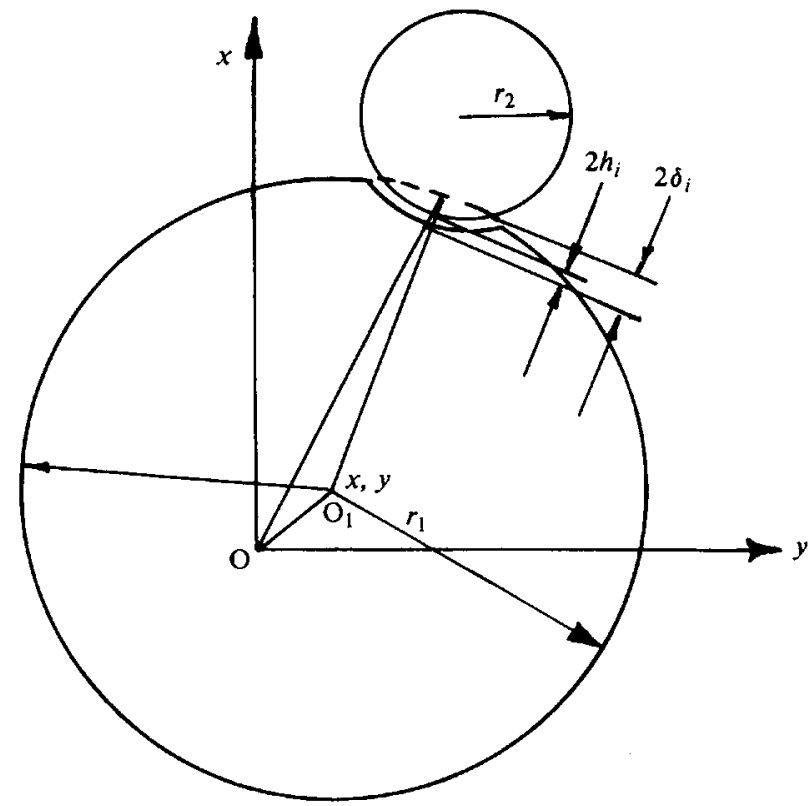

Fig. 1 Bearing geometry

completely rigid body assumpion makes both speeds the same. Expressed mathematically, the elastic body assumption is as follows. Referring to Fig. 1, the film thickness equation is

$$
h(x, y)=h(0,0)+\delta(x, y)-\delta(0,0)+P_{r}(x, y)
$$

where $P_{r}(x, y)$ is the local undistorted film profile and does not alter with time, $\delta(x, y)$ is the surface deformation relative to the element centre, which is considered remote and has only a rigid body movement, and $\delta(0,0)$ is the surface deflection at the lubricated contact centre. Differentiating equation (1) with respect to time,

$$
\dot{h}(x, y)=\dot{h}(0,0)+\dot{\delta}(x, y)-\dot{\delta}(0,0)
$$

The rigid body assumption makes $\dot{h}(x, y)=\dot{h}(0,0)$, and $\delta(x, y)=\delta(0,0)=0$. An elastic surface having a uniform squeeze velocity makes $\dot{h}(x, y)=\dot{h}(0,0)$ and $\dot{\delta}(x, y)=$ $\delta(0,0)$, though each relative deformation rate does not necessarily equal zero. Mostofi (8) has solved numerically the e.h.l. problem assuming the usual external conditions of rolling speed, load, oil properties, geometry and a fully flooded inlet, but with additionally a uniform surface squeeze speed on the RHS of Reynolds' equation. He obtained a regression formula for the least film thickness in an elliptical contact in terms of all these external parameters. In terms of $W^{*}$ (the integrated dimensionless pressure), it comes to

$$
W^{*}=K^{\prime} \frac{\left(1-A_{i}^{*)^{\prime}}\right.}{h_{i}^{\alpha^{* *}}}
$$

where

$$
\begin{aligned}
\alpha^{\prime}= & 22.3 \\
A_{i}^{*}= & A_{0} \mathrm{e}^{\beta w_{s i}} \\
\beta= & \frac{132}{U} \\
A_{0}= & 0.75 \\
K^{\prime}= & 3.206\left(U^{*}\right)^{14.43}\left(G^{*}\right)^{10.25} \\
& \times\left(1-0.683 \mathrm{e}^{-0.669} e_{p}^{*}\right)^{22.3} \times 10^{25}
\end{aligned}
$$




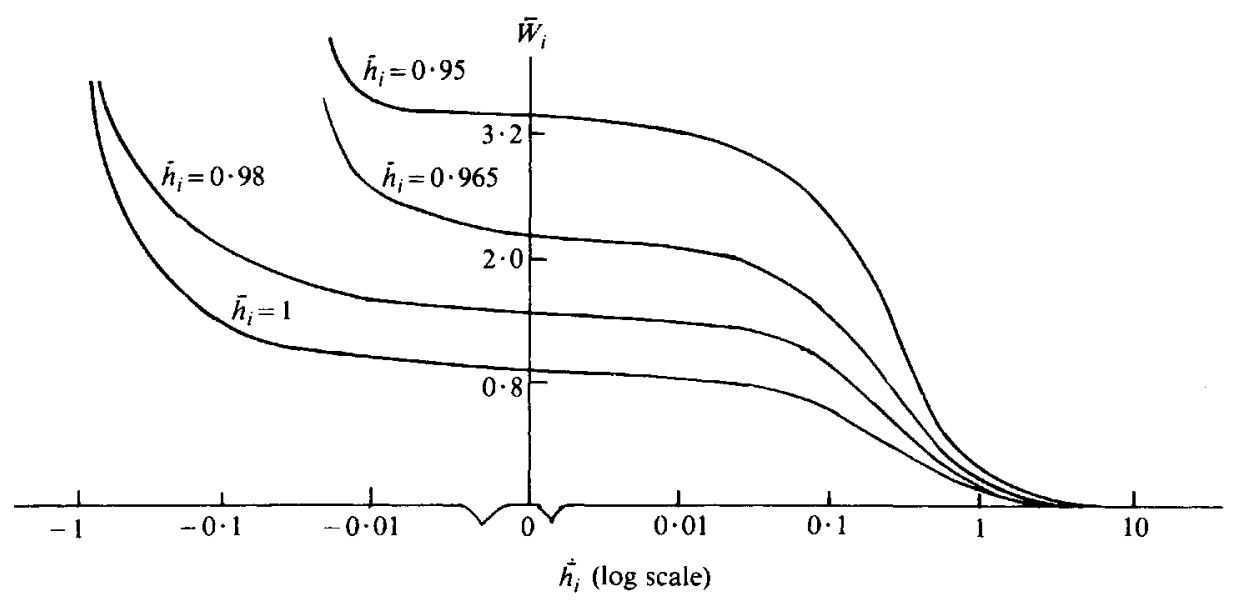

Fig. 2 Variation of load with film thickness and squeeze speed

A similar expression is found in (9), though without the squeeze term. The various dimensionless groups are defined in the notation. The sign convention in Fig. 1 makes $w_{s i}^{*}$ negative in equation (2) if ball and race contact surfaces are approaching one another. Thus $W^{*}$ increases for a constant $h_{i}^{*}$, though clearly, in the actual dynamical problem we have here, $h_{i}^{*}$ would generally decrease as well. Equation (2) only strictly applies for the range of external parameters used in the computations of $(\mathbf{8}, 9)$, but is employed in the analysis below over a much wider range for want of a more comprehensive formula. For $w_{s i}^{*}$ (see notation) increasing negatively, equation (2) is well behaved, but for $w_{s i}^{*}$ positive (surfaces separating) its range is limited because $\left(1-0.75 \mathrm{e}^{132 w_{\mathrm{s} i} / U}\right)$ can become a negative. (When $w_{\mathrm{s} i}^{*}=$ 0 , we have pure rolling motion.) In a vibrating ball bearing, all regimes of lubrication may be encountered. Thus, elements can successively be under hydrodynamic, piezoviscous and e.h.l. conditions, with the squeeze velocity being of either sign, making a comprehensive formula for the instant surface load under an element (i) necessary.

Assuming that the dynamics of the shaft are influenced mainly by the e.h.l. pressure contribution, and neglecting the piezoviscous zone, a general approximate expession for the instant surface load can be written. This is cast in normalized form, as it eliminates the large and small quantities found in equation (2) and is therefore better suited for subsequent computations. It comes to

$$
\bar{W}_{i}=\Psi_{1}\left(\frac{1-\bar{A}_{i}}{\bar{h}_{i}}\right)^{\alpha^{\prime}}+\Psi_{2}\left(\frac{1-\bar{B}_{i}}{\bar{h}_{i}}\right)^{1 / 2}
$$

where

$$
\begin{gathered}
\bar{W}_{i}=\frac{W_{i}^{*}}{W_{0}^{2}}, \quad \Psi_{1}=\frac{K^{\prime}}{W_{0}^{*}\left(h_{0}^{*}\right)^{\alpha^{\prime}}}, \quad \Psi_{2}=\frac{C_{\text {iso }}}{W_{0}^{*}\left(h_{0}^{*}\right)^{1 / 2}} \\
\bar{A}_{i}=A_{0} \mathrm{e}^{\bar{\beta} \bar{h}_{i}}, \quad \bar{\beta}=\beta \tau h_{0}, \quad \beta=\frac{132}{U}, \quad A_{0}=0.75 \\
\bar{B}_{i}=B_{0} \mathrm{e}^{\bar{\lambda}_{i}}, \quad \bar{\lambda}=\lambda \tau h_{0}, \quad \lambda=\frac{67.7}{U}, \quad B_{0}=0.75 \\
\bar{h}_{i}=\frac{\bar{W}_{s i}}{\tau h_{0}}, \quad C_{\text {iso }}=\frac{24 \pi U^{*}}{\sqrt{\left(2 \bar{R} h_{0}^{*}\right) 2(3+2 \bar{R})}}
\end{gathered}
$$

(C) IMechE 1985 $\tau$ is a time scaling factor, $W_{0}^{*}$ and $h_{0}^{*}$ are respectively the dimensionless load and film thickness at time $t=0$, and other terms are defined in the notation.

The second term on the RHS of equation (3) is the isoviscous contribution to the instantaneous load. When it is relatively large, the e.h.l. term is negligible and even negative and vice versa. It is an approximation to an exact expression which is derived in Appendix 1.

The way $\bar{W}_{i}$ varies with $\dot{\bar{h}}_{i}$ and $\bar{h}_{i}$ is shown in Fig. 2. When $\bar{h}_{i}$ becomes large and positive, $\bar{W}_{i}$ can go negative in equation (3); to ensure it will remain positive under all conditions at $\bar{B}_{i}=1$, the second term in equation ( 3 ) is replaced by an approximation of the form $C_{3} / h_{i}+C_{2}$, where $C_{3}$ and $C_{2}$ are derived in Appendix 2. Thus, the balls always push inwards on the shaft through their lubricant films, though often these forces are negligible.

\subsubsection{Model geometry}

Consider the balls and the outer race all to be rigid, having a radial clearance $2 C$ with the shaft. For purposes of computation, let the inner race have a reduced modulus, with all the elastic distortion occurring on its surface as the elements roll over it with a centre-line film thickness $2 h(0,0)$ and deflection $2 \delta(0,0)$. Then for a shaft centre displacement $x, y$ from the axial symmetry position ( $z$ axis), and considering the $i$ th ball,

$$
2 \delta(0,0)=2\{h(0,0)-C\}+x \cos \theta_{i}+y \sin \theta_{i}
$$

Now equations (1) and (2) are based on the minimum oil film thickness near the ends of the long axis of the elastostatic contact ellipse. Furthermore, the expression for $h^{*}(0,0)$ in (8) gives an index for $W^{*}$ of +0.004 because of its extreme insensitivity to load. The same effect was noted in (10). Generally, the ratio of centreline to minimum oil film thickness in e.h.l. contacts does not exceed $1.3(9)$, so $h(0,0)$ can be replaced by $h_{i}$ in equation (3) without much error being introduced when eventually considering the system dynamic behaviour. $\delta(0,0)$ is retained because a further simplifying assumption is made later with respect to it. Equation (4) therefore becomes, for the ith ball,

$$
2 \delta_{i}=2\left(h_{i}-C\right)+x \cos \theta_{i}+y \sin \theta_{i}
$$
where $\delta_{i}=\delta(0,0)$ and $h_{i}$ is the minimum oil film thickness. 
If there is instead a radial interference fit $\rho, C$ is replaced by $-\rho$ in equation (4).

The initial conditions assume axial symmetry about the $z$ axis by holding the shaft centre at $\mathrm{O}(x=y=0)$ until $t=0$ and then releasing it. Thus, initially each rotating ball in the set has the same film thickness $2 h_{0}$ and deflection $2 \delta_{0}$ under it, making equation (5)

$$
\delta_{0}=h_{0}+\rho
$$

Using $\delta_{0}$ and $h_{0}$ as scaling factors (see notation) equation (4) becomes

$$
2 \bar{\delta}_{i}=2\left(\bar{h}_{0} \bar{h}_{i}+\bar{\rho}\right)+\bar{x} \cos \theta_{i}+\bar{y} \sin \theta_{i}
$$

\subsubsection{Equations of motion}

Assuming the balls have negligible mass and an external force vector having components $F_{x}$ and $F_{y}$ acts in the plane of $x y$, as in Fig. 3, the equations of motion are

$$
\begin{aligned}
& M \ddot{x}=\left(\sum_{i=1}^{m}-W_{i} \cos \theta_{i}\right)-M g+F_{x} \\
& M \ddot{y}=\left(\sum_{i=1}^{m}-W_{i} \sin \theta_{i}\right)+F_{y}
\end{aligned}
$$

if

$$
\begin{aligned}
\bar{P}^{\prime} & =\frac{W_{0}}{M \tau^{2} \delta_{0}}, \quad \ddot{\bar{x}}=\frac{\ddot{x}}{\delta_{0} \tau^{2}}, \quad \ddot{\bar{y}}=\frac{\ddot{y}}{\delta_{0} \tau^{2}} \\
\vec{F}_{\bar{x}} & =\frac{F_{x}}{W_{0}}, \quad \bar{W}_{i}=\frac{W_{i}}{W_{0}} \\
\bar{F}_{\bar{y}} & =\frac{F_{y}}{W_{0}} \\
\ddot{\bar{x}} & =-\bar{P}^{\prime}\left(\sum_{i=1}^{m} \bar{W}_{i} \cos \theta_{i}\right)-\frac{M g}{W_{0}}+\bar{F}_{\bar{x}} \\
\ddot{\bar{y}} & =-\bar{P}^{\prime}\left(\sum_{i=1}^{m} \bar{W}_{i} \sin \theta_{i}\right)+\bar{F}_{\bar{y}}
\end{aligned}
$$

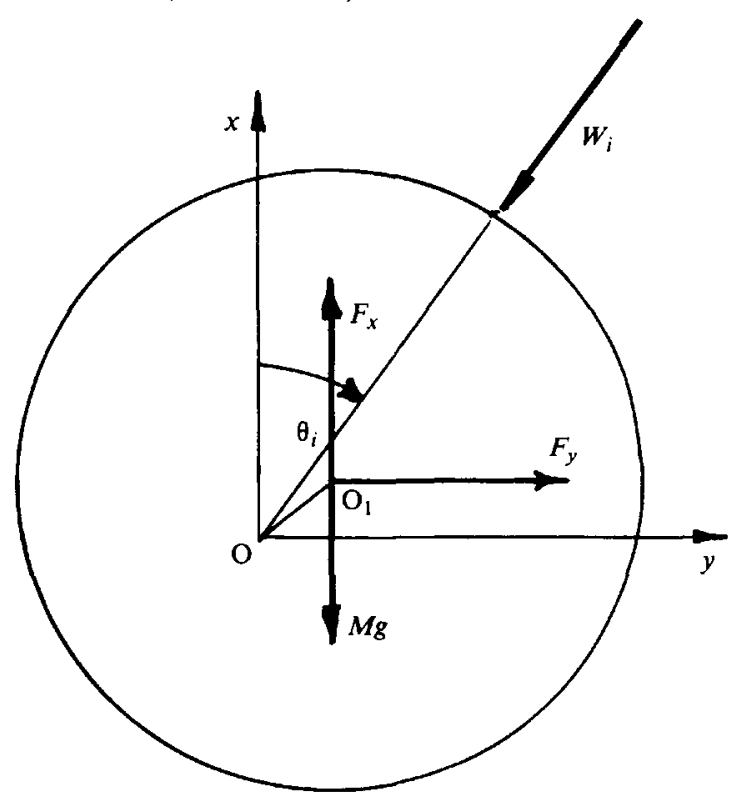

Fig. 3 Forces on the inner race
The value of the time scaling factor $\tau$ is set for convenience by making $\bar{P}^{\prime}=1$, giving

$$
\tau=\left(\frac{W_{0}}{M \delta_{0}}\right)^{1 / 2}
$$

Also,

$$
\theta_{i}=\theta_{0 i}+\omega_{c} t
$$

or

$$
\theta_{i}=\frac{2 \pi i}{m}+\omega_{c} t
$$

where

$$
i=1, m \quad \text { and } \quad \omega_{c}=\frac{\omega_{1} r_{1}}{2\left(r_{1}+r_{2}\right)}
$$

\subsection{Elastic deflection}

With the shaft rotating at speed $\omega_{1}$ and its centre initially at $x=y=0$, let $m$ equal equipitched forces $W_{0}$, rotating at speed $\omega_{\mathrm{c}}=\dot{\theta}_{i}$, act radially inwards on its circumference. For the purposes of computation, the shaft can now be considered as not rotating, but having the $W_{0}$, and afterwards the $W_{i}$, forces rotating round it at the cage speed $\dot{\theta}_{i}$, with $F_{x}$ and $F_{y}$ acting in some prescribed manner which may be a function of its rotational speed (such as a rotating unbalance). An additional governing equation is necessary for the computations. This is the relationship between $W_{i}$ and $\delta_{i}$ in equation (5). As $\delta_{i}$ is at each contact centre, and the oil film is considered parallel and equal to $h_{i}$ when conditions are e.h.l., then

$$
\bar{W}_{i}=\frac{k \delta_{0}^{\gamma}}{W_{0}} \bar{\delta}_{i}^{\gamma}
$$

where $\gamma=3 / 2$ and $\bar{\delta}_{i}$ is the elastostatic deflection at the centre of an elliptical footprint (11). The initial conditions can now be calculated. If $U$ is sufficiently high (which it usually is), the conditions are e.h.l. everywhere, so equation (3) becomes

$$
W_{0}^{*}\left(h_{0}^{*}\right)^{\alpha^{\prime}}=K^{\prime}
$$

Combining equation (11) with equations (6) and (10) under initial conditions, allows $W_{0}, h_{0}$ and $\delta_{0}$ to be found.

\subsubsection{Calculation procedure}

\section{Find the model constants}

1. Choose the bearing geometry, radial clearance or interference fit, shaft rotational speed, number of balls, oil and material properties, shaft mass and excitation force function.

Set the initial conditions

2. At $\bar{t}=0-$, calculate $W_{0}, h_{0}$ and $\delta_{0}$ from equations (6), (10) and (11).

\section{Motion commences}

3. At $\bar{t}=0+$ (the moment of release), calculate $\ddot{\bar{x}}(0)$ and $\ddot{\bar{y}}(0)$ from equations $(8)$ and $(9)$.

4. Differentiate equations (3) and (10) with respect to $\bar{t}$ and equate them at $\bar{t}=0$, to find $\ddot{\bar{s}}_{i}(0)=\bar{h}_{i}(0)$. 


\section{The iteration procedure commences}

5. Using the average acceleration quasi-dynamic procedure (12) (see Appendix 3) find

$$
\dot{\bar{x}}, \dot{\bar{y}}, \bar{x}, \bar{y}, \bar{h}_{i}, \dot{\bar{S}}_{i}, \ddot{\bar{S}}_{i} \quad \text { for } i=1, m
$$

where

$$
\theta_{i}=i \frac{2 \pi}{m}+\bar{\omega}_{\mathrm{c}} \bar{t}
$$

$\bar{S}_{i}=\bar{h}_{i}-1$ and is the ripple on the initial film thickness $h_{0}$

6. Calculate $\bar{\delta}_{i}$ and $\dot{\bar{\delta}}_{i}$ from equation (7) and its differential with respect to $\bar{t}$.

7. Calculate $\bar{W}_{i}$ from equation (3) or its replacement in Appendix 2 (depending on its location in Fig. 2).

8. Calculate $\hat{S}_{i}$ at $\bar{t}=k \delta \bar{t}$ where $k=1, n$ and $n=$ number of time steps used.

9. Find $\ddot{\bar{x}}(\bar{t})$ and $\ddot{\bar{y}}(\bar{t})$ from equations (8) and (9).

10. Test for convergence using the convergence criteria

$$
\frac{\ddot{\bar{x}}_{k, j}-\ddot{\bar{x}}_{k,(j-1)}}{\overline{\bar{x}}_{k, j}} \leqslant \varepsilon_{\bar{x}}
$$

and

$$
\frac{\ddot{\bar{y}}_{k, j}-\ddot{\bar{y}}_{k,(j-1)}}{\ddot{\bar{y}}_{k, j}} \leqslant \varepsilon_{\bar{y}}
$$

where $j=$ iteration counter within each time step $k$.

11. If converged, increment $\bar{t}$ to $2 \delta \bar{t}$ and return to stage 5 .

If not converged return to stage 5 but with $k$ unchanged.
12. Proceed until step 10 is satisfied and increment further the time.

\subsubsection{Particulars of the system model}

$\begin{array}{ll}\text { Inner race bore } & 40 \mathrm{~mm} \\ \text { Inner race diameter } & 50 \mathrm{~mm} \\ \text { Outer race diameter } & 75.4 \mathrm{~mm} \\ \text { Ball diameter } & 12.7 \mathrm{~mm} \\ \text { Outer race outside diameter } & 83.7 \mathrm{~mm} \\ \text { Bearing width } & 23.4 \mathrm{~mm} \\ \text { Number of balls (generally) } & 12 \\ \text { Ellipticity of elastostatic footprint } & 3\end{array}$

\subsubsection{Results for a gravity load application}

The shaft, previously held centrally at $t=0$, is released under its own weight, thus subjecting the system to a step gravity load. As all bearing surfaces are perfect here, the subsequent movement is known as a variable compliance vibration.

Figure 4 shows the resulting shaft centre-line time history for the model subjected to the following conditions:

$$
\begin{array}{ll}
\text { Shaft rotational speed } & 209 \mathrm{rad} / \mathrm{s}(1995 \mathrm{r} / \mathrm{min}) \\
\text { Cage set speed } & 83.26 \mathrm{rad} / \mathrm{s} \\
\text { Step load, } Q=\mathrm{Mg} & 500 \mathrm{~N} \\
\text { Radial interference } & 2 \rho=10 \mu \mathrm{m} \\
\text { Number of balls } & m=12
\end{array}
$$

The oscillations commence at $x=y=0$. In Fig. $4 a$, the $x$ displacement component in the direction of gravity initially has an oscillatory decay about the equilibrium

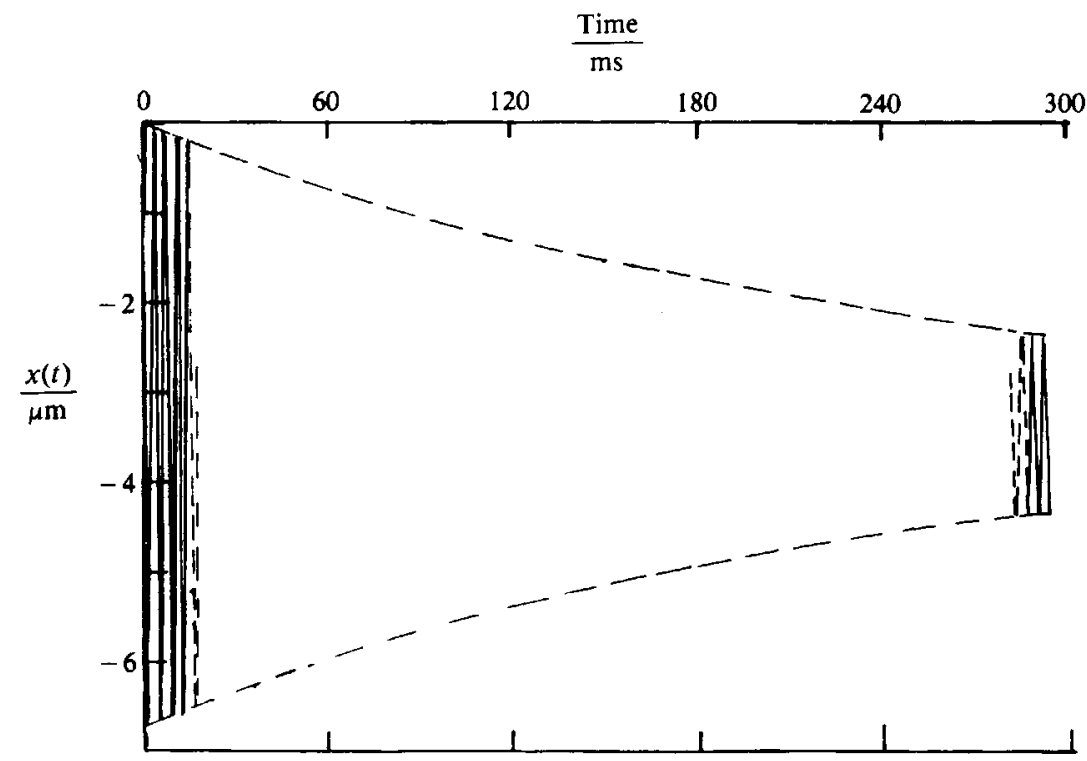

(a) $x$ component

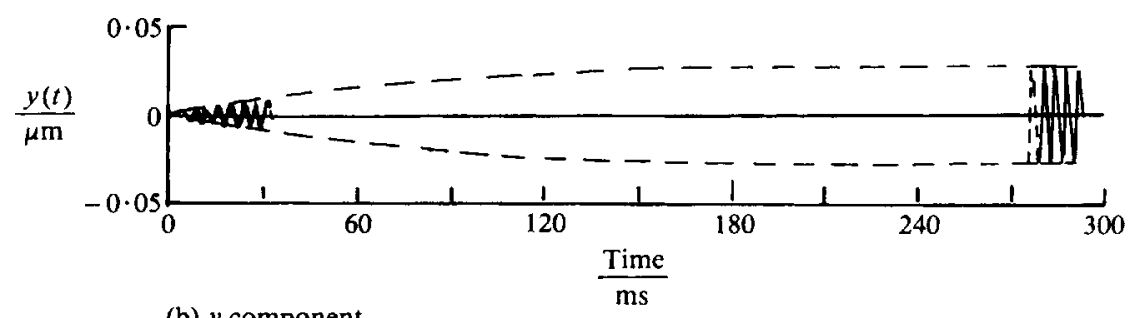

(b) $y$ component

Fig. 4 Shaft centre time history $(Q=500 N, \rho=5 \mu \mathrm{m}, m=12)$ 
position (of about $x=3.5 \mu \mathrm{m}$ ) and about which it finally goes into a limit cycle, this being a characteristic of non-linear vibratory systems. The cycle is maintained because an external energy source (the shaft drive motor) has to rotate the cage while its ball complement is being squeezed and released in their oil films as they continually change position. The limit cycle vibration double amplitude in the $x$ direction is only about $2 \mu \mathrm{m}$. In the $y$ direction (Fig. 4b), the vibrations build up from zero amplitude to a small limit cycle with a double amplitude of $0.05 \mu \mathrm{m}$. Observe that it takes $300 \mathrm{~ms}$ to reach a steady state vibration (when transients have subsided), during which time the shaft has made about 20 revolutions. The measured response frequency of the system is $260 \mathrm{~Hz}$, which is marginally less than the undamped frequency, found independently (13), and differs from the calculated ball pass frequency of $157.1 \mathrm{~Hz}$.

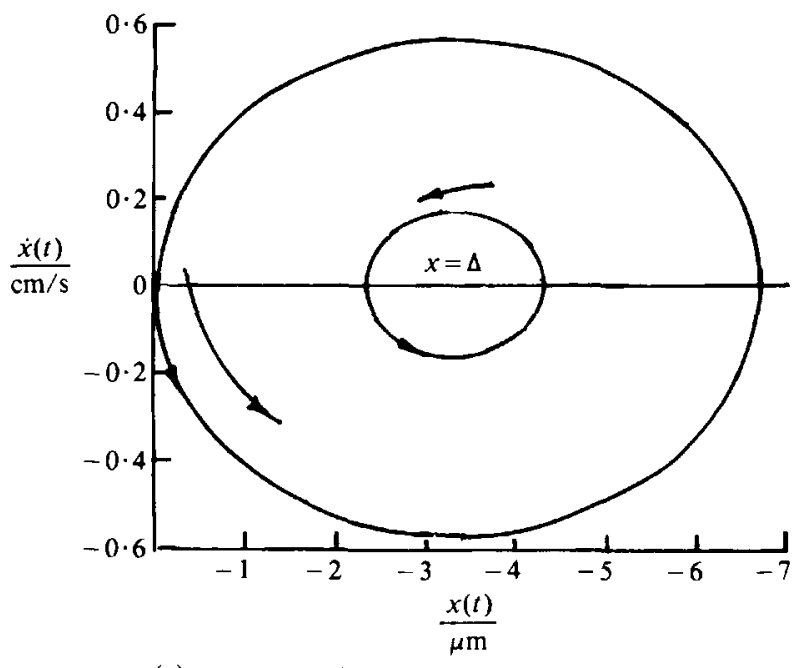

(a) $x$ component

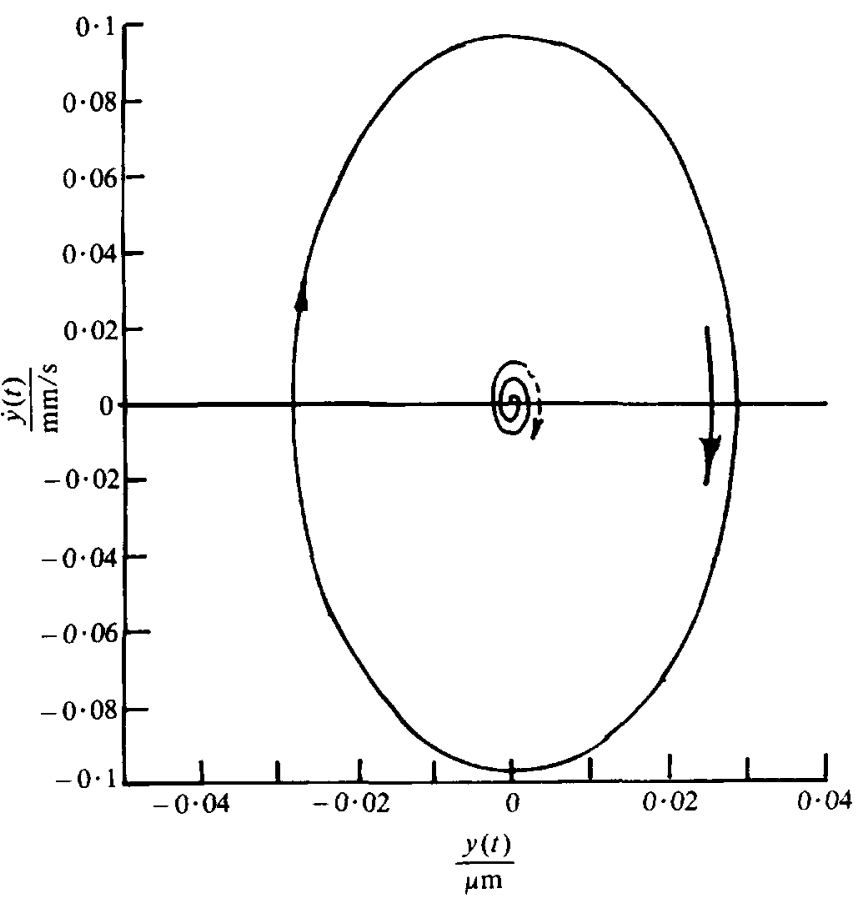

(b) $y$ component

Fig. 5 Phase plane trajectories of shaft centre $(Q=500 \mathrm{~N}$, $\rho=5 \mu \mathrm{m}, m=12$ )
When the $x$ and $y$ oscillations are plotted as trajectories in the phase plane, namely $x-\dot{x}$ and $y-\dot{y}$, as shown in Figs $5 a$ and $5 b$, the $x-\dot{x}$ curve spirals in towards an elliptically shaped stable limit cycle (14) with its centre on the equilibrium position. The time for one rotation round the limit cycle path is again the response frequency of the system. This appears to be the base frequency of the non-linear system. A detailed frequency analysis of the centre movement would probably yield a series of harmonics in addition to the base frequency. One way of doing this would be to subject the bearing to a Dirac function pulse at $t=0$ and then use fast Fourier transforms to analyse the harmonic content of the response. References (13) and (14) discuss this method of frequency analysis, which is used below in the analysis of the response to a wavy inner race surface. When $x$ is plotted against $y$, the shaft centre locus is obtained. Figure 6 shows the effect of radial interference $\rho$ on the locus, and its eventual limit cycle. Figure $6 \mathrm{a}$ is for $\rho=5 \mu \mathrm{m}$. Only the initial part of the locus is shown, together with the limit cycle, which corresponds to the phase plane representation in Fig. 5. When $\rho$ is reduced to $1 \mu \mathrm{m}$, the limit cycle is centred about a larger $x$ component equilibrium position and also has larger $x$ and $y$ amplitudes. This is because the reduced radial interference makes the balls and races operate in a less stiff region of the non-linear loaddeflection curve, the response frequency now being $125 \mathrm{~Hz}$. Furthermore, the angular disposition of the limit cycle has altered, suggesting that the reduced interference makes squeeze film damping more effective, thus altering the phase angle between $x$ and $y(\mathbf{1 5})$. Note also that the limit cycle in Fig. $6 \mathrm{~b}$ is centred slightly to the left of the $y=0$ axis. The reason is that as the cage set rotates clockwise relative to a stationary observer, the elements in the bottom right quadrant of the shaft will suffer the highest squeeze during any cycle, unless the shaft moves to the left to compensate. When the shaft rotation vector is reversed, the limit cycle centre moves to the right of the $y=0$ axis.

The steady state lubricant film history for one ball can also be shown in the phase plane by phase trajectories of $h-\dot{h}$, as shown in Fig. 7 . It is composed of two unequal portions, of similar shape, with a common point $A$. The larger segment represents film behaviour when the ball is in the unloaded region of the bearing, where there is a significant hydrodynamic contribution. The smaller segment, to the left of $A$, corresponds to the more highly loaded region which is the elastohydrodynamic part. Of course, each region does not necessarily apply always to the top or bottom of the shaft, as its centre is itself oscillating. A limit cycle period corresponds to oscillations which commence and terminate at $\mathrm{A}$, describing all the trajectory loops in a to-and-fro motion as outlined below.

One film limit cycle goes from the outer to the inner loops of the $\mathrm{RH}$ segment clockwise, followed by the inner to outer loops of the LH segment anticlockwise. It then repeats the operation in a total time $T_{c}$, where $T_{c}=2 \pi / \omega_{c}$ and $\omega_{c}$ is the cage set rotational speed. Any one loop traverse is the system response frequency $1 / T$, where $T=T_{c} / 20$ is also the time for one traverse of the $x-\dot{x}$ limit cycle in Fig. 5a. Note that as the loops on the RH side of point $\mathrm{A}$ enclose larger areas than those on the LH side, there is more energy dissipation associ- 

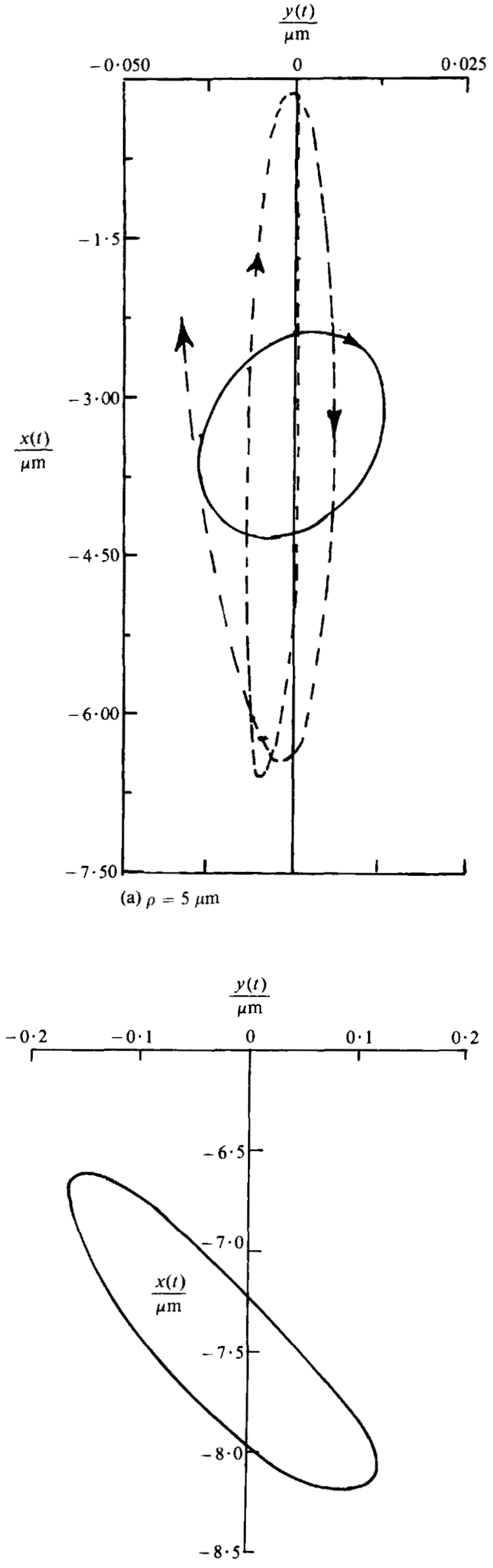

(b) $\rho=1 \mu \mathrm{m}$

Fig. 6 Limit cycle shaft centre $(Q=500 \mathrm{~N}, m=12)$

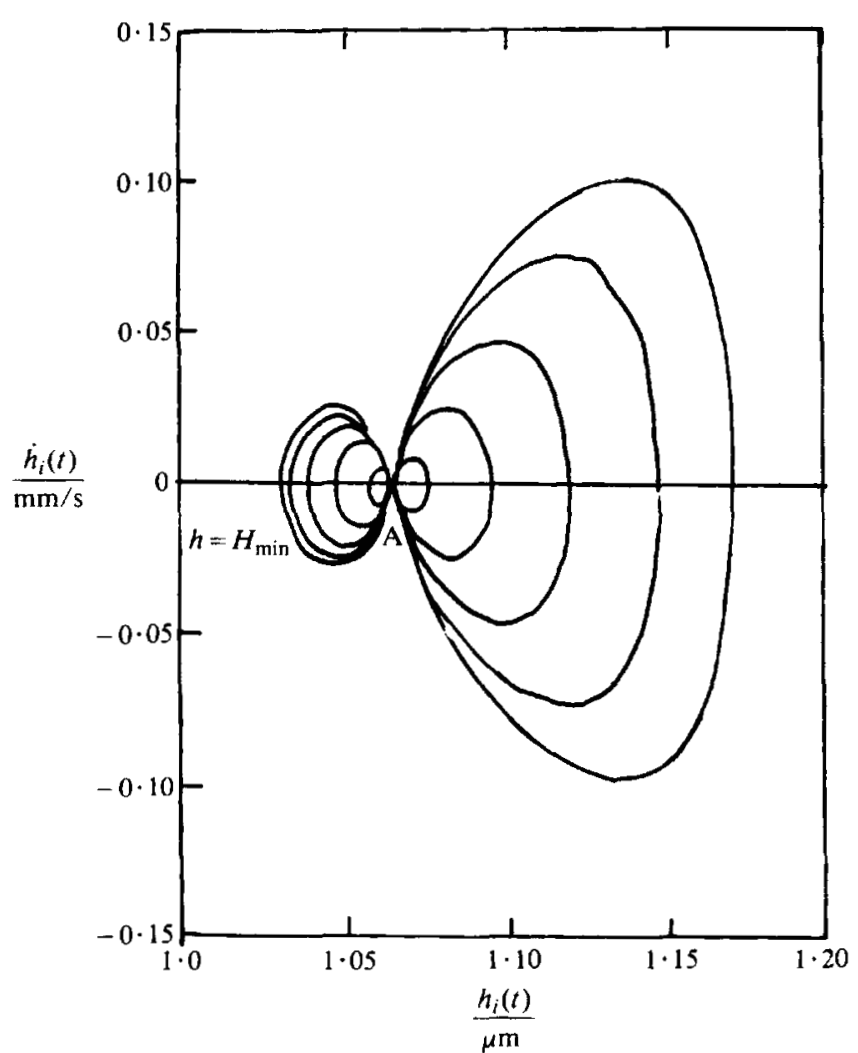

Fig. 7 Limit cycle phase plane trajectory of oil film $(Q=$ $500 \mathrm{~N}, \rho=5 \mu \mathrm{m}, m=12$ )

ated with them, suggesting that squeeze film damping operates most efficiently in the region of thicker films.

The periodic load experienced by a single ball during a complete revolution of the cage is shown in Fig. 8. The cyclic load distribution comprises 20 oscillations corresponding to the 20 loops in the $h-\dot{h}$ limit cycle. Points $A_{1}$ and $A_{3}$ relate the ball inlet to, and exit from, the most loaded region of the bearing (the small segment of the $h-\dot{h}$ limit cycle). The curve following the mean of the natural frequency oscillations (the carrier) is itself oscillatory at the cage set frequency and has a mean value determined by the preload. It represents the average load on a ball during one revolution of the cage. Clearly, the superimposed vibration must adversely affect the fatigue life of the bearing. If preload

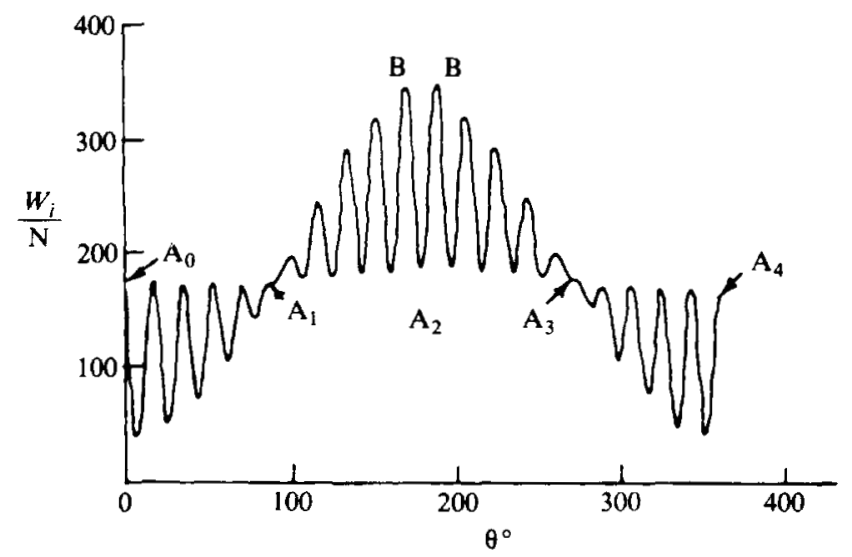

Fig. 8 Cyclic load distribution on a ball $(Q=500 \mathrm{~N}, \rho=$ $5 \mu \mathrm{m}, m=12$ ) 
exactly equalled zero, then the average load curve would oscillate about the zero axis, cauing the ball to be unloaded over half a cycle. Such a state of affairs is associated with the unloaded balls skidding on the races, thus causing damage to them and the cage. Radial preload (normally created by axially loading the balls) goes some way to curing the problem (5).

\subsubsection{Squeeze film damping}

Oil film damping can be studied upon the application of a step gravity load. The degree of damping is measured by the rate of oscillation decay to the limit cycle in Fig. 4. The damping is described by the logarithmic decrement, $d$, of amplitude peak decay. Therefore,

$$
d=\ln \left(\frac{x_{n}}{x_{n+1}}\right)
$$

and for small damping, as we have here (12),

$$
\frac{x_{n+1}}{x_{n}}=\mathrm{e}^{-d} \simeq 1-d
$$

Thus:

$$
d=\frac{x_{n}-x_{n+1}}{x_{n}}
$$

and the damping ratio is

$$
\zeta=\frac{d}{2 \pi}
$$

Figure 9 shows how $\zeta$ varies with $\bar{\rho}$ for two different loads. Point $\mathbf{P}$ on the graph corresponds to $\bar{\rho}=1$. As the initial film thickness (at $t=0$ ) is given by $h_{0}=\delta_{0}$ $-\rho$, that is $\bar{h}_{0}=h_{0} / \delta_{0}=1-\bar{\rho}, h_{0}=0$ there, which is the hypothetical condition of zero film thickness. There is no damping under this condition. The higher applied load gives a larger damping coefficient because of the larger films and squeeze velocities formed in the top half of the bearing. Dareing and Johnson (2) have come to a similar conclusion when they carried out experiments on loaded discs, one of which was corrugated.

The influence of the number of balls on the response frequency is shown in Fig. 10. Increasing the number of balls $m$ stiffens the bearing. For any given value of $m$, the higher load gives a lower response frequency despite

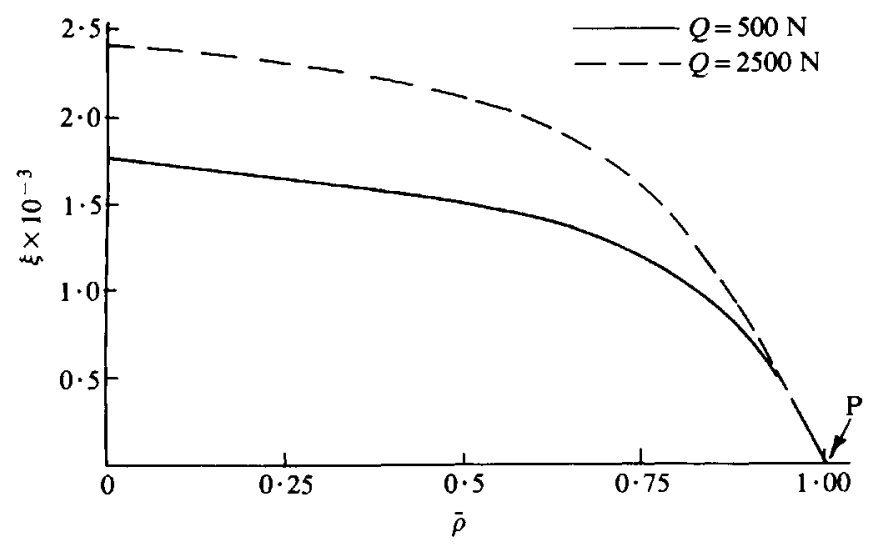

Fig. 9 Influence of radial interference on the damping factor $(m=12)$

Proc Instn Mech Engrs Vol 199 No C3

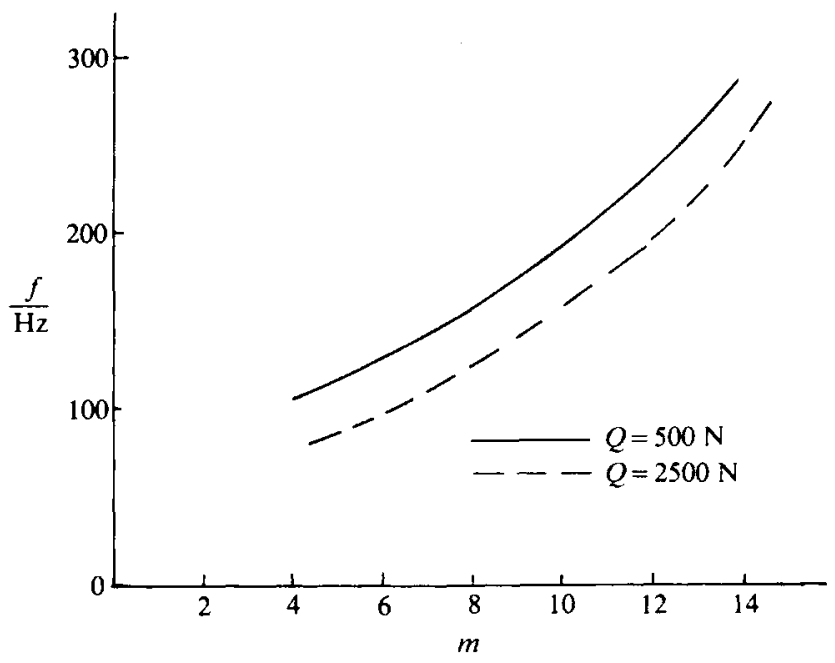

Fig. 10 Influence of the number of balls on the natural frequency $(\bar{\rho}=0.75)$

some balls entering a more stiff region of their loaddeflection characteristics. The effect of $m$ on the damping coefficient is shown in Fig. 11. Increasing the number of balls stiffens the bearing and reduces the film thicknesses in the unloaded region, thereby reducing the damping coefficient. A higher load increases these films for a given ball complement, thereby increasing the damping coefficient.

\subsubsection{Vibrations of an unbalanced shaft}

If the supported shaft's centre of gravity is given a slight eccentricity $e$, the external exciting force components in equations (8) and (9) become

$$
\bar{F}_{\bar{x}}=\frac{M \omega_{1}^{2}}{W_{0}} e \cos \theta_{i} \quad \text { and } \quad \bar{F}_{\bar{y}}=\frac{M \omega_{1}^{2}}{W_{0}} e \sin \theta_{i}
$$

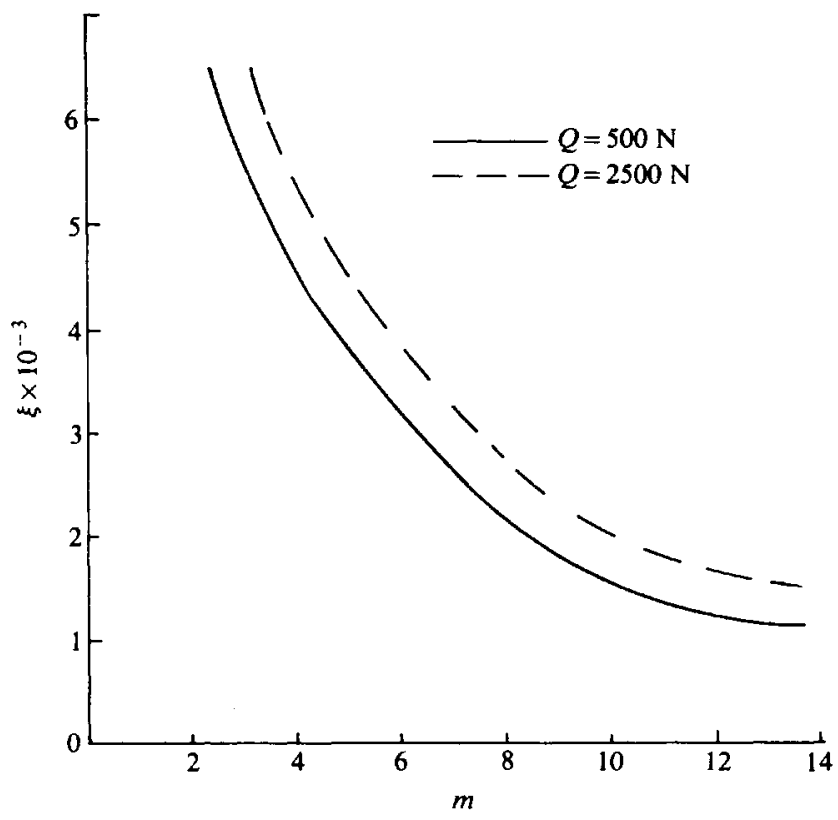

Fig. 11 Influence of the number of balls on the damping coefficient $(\bar{\rho}=0.75)$ 


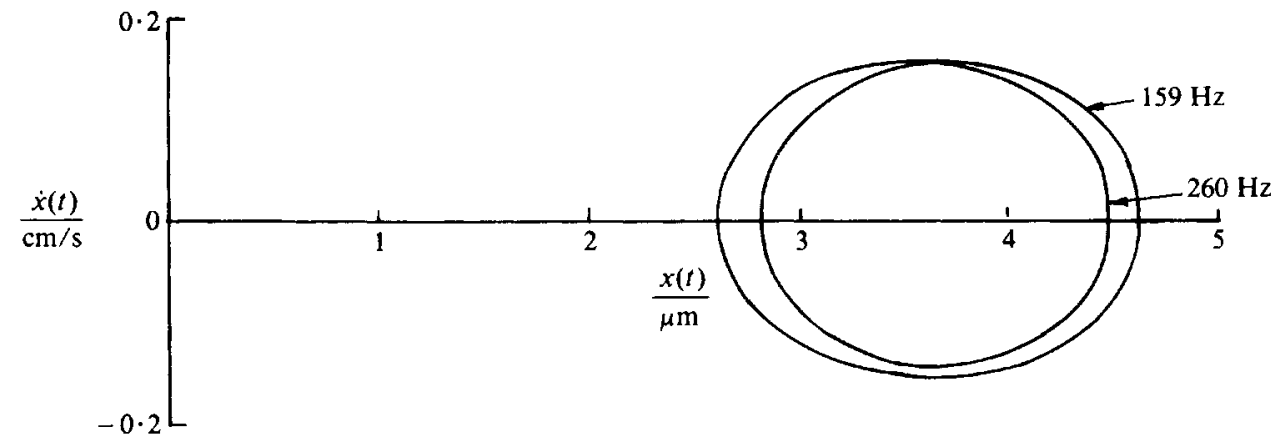

Fig. 12 Steady state phase plane $x$ component trajectory with rotating unbalance $\left(Q=500 \mathrm{~N}, \omega_{1}=209 \mathrm{rad} / \mathrm{s}, e=25 \mu \mathrm{m}, \rho=5 \mu \mathrm{m}\right.$, $m=12$ )

The resulting limit cycle in the phase plane, for the $x$ oscillation, is shown in Fig. 12. The double loop indicates a response dominated by two frequencies forming upper and lower bounds. The inner loop is the model natural frequency and the outer loop coincides with the ball-pass frequency, which is here defined as $m \omega_{1} r_{1} /\left\{2\left(r_{1}+r_{2}\right)\right\}$. Experiments carried out by Wardle and Poon (1) on angular contact bearings have come to a similar conclusion. The corresponding $x-y$ locus, in the steady state, is shown in Fig. 13. The complex motion reveals the various controlling frequencies involved. Note that the $y$ displacement is now significant because of the rotating unbalance.

\subsubsection{Rolling surface imperfections}

The quality of a rolling bearing is described by the finish and form of its surfaces. These distributed features are generated during manufacture and are usually defined in terms of their wavelength. If the wavelength of the surface irregularities exceeds the width of the elastostatic contact footprint in the rolling direction, the feature is termed waviness (1); otherwise it is termed roughness. We confine ourselves here to waviness.

Let the inner race surface have all the waviness in the form of a circumferential sinusoidal wave. The contact radial clearance becomes now a function of angular position $\theta^{\prime}$. If $2 C$ is the local clearance at $\theta^{\prime}$,

$$
2 C=2 C_{0}+2 C_{p} \sin \frac{2 \pi r_{1} \theta^{\prime}}{\lambda_{1}}
$$

where $C_{p}$ is the wave amplitude and $\lambda_{1}$ the wavelength. If $C_{0}=0$, both radial interference and clearance occur alternately round the race. To ensure interference everywhere, replace $2 C_{0}$ in equation (5) by $-2 \rho_{0}$. Thus:

$$
2 \rho=2 \rho_{0}+2 \sin \left(\frac{2 \pi}{\lambda_{1}} r_{1} \theta^{\prime}\right)
$$

where $\rho_{p}$ is the wave amplitude associated with an interference fit. If there are $n$ waves round the circumference,

$$
\lambda_{1}=\frac{2 \pi r_{1}}{n}
$$

The points of interest are when $\theta^{\prime}=\theta_{i}$, so the radial interference at the $i$ th contact is non-dimensionally

$$
2 \bar{\rho}_{i}=2 \bar{\rho}_{0}+2 \bar{\rho}_{p} \sin \left(n \theta_{i}\right)
$$

where

$$
\bar{\rho}_{i}=\frac{\rho_{i}}{\delta_{0 i}} \text { and } \bar{\rho}_{p}=\frac{\rho_{p}}{\delta_{0}}
$$

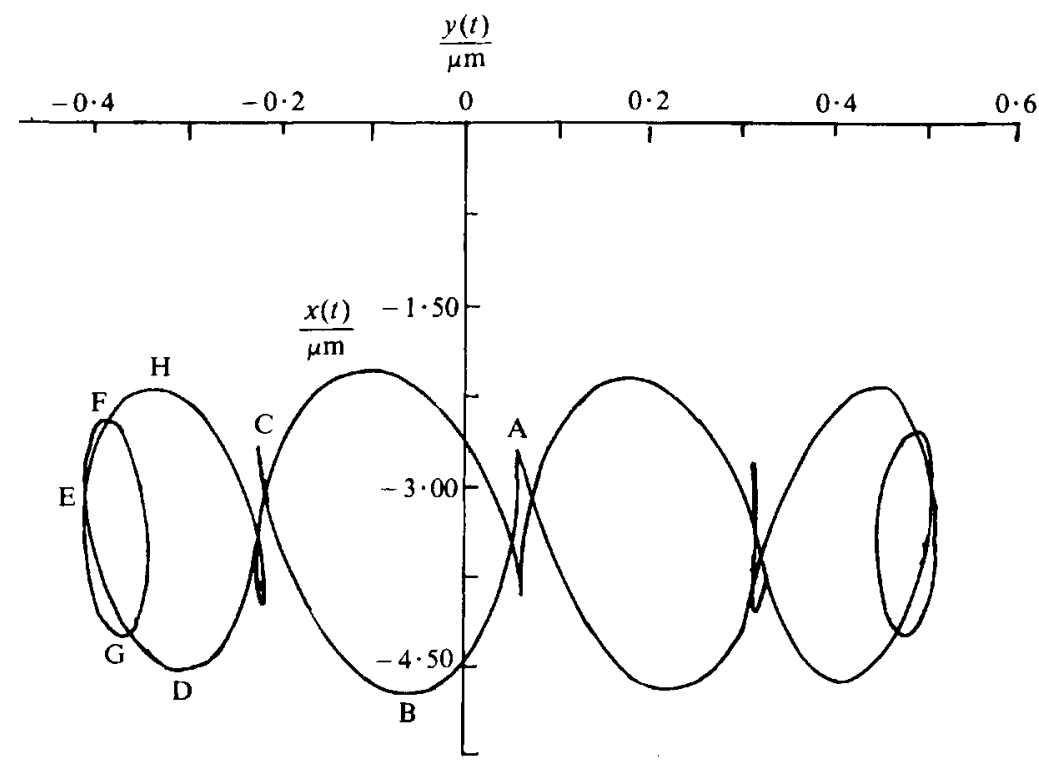

Fig. 13 Shaft centre locus, steady state $\left(Q=500 \mathrm{~N}, \omega_{1}=209 \mathrm{rad} / \mathrm{s}, e=25 \mu \mathrm{m}, \rho=5 \mu \mathrm{m}, m=12\right)$ 
To reduce computing space allocation, let $\delta_{0 i}=\delta_{0}$, the universal initial contact deflection. Therefore there are no surface features at $t=0$ until they suddenly appear at $t=d t$ where $d t \ll 1 \mathrm{~ms}$. The governing equations in the elastohydrodynamic model remain unaltered except for those describing the local rate of deformation when $\bar{t}>0$. Differentiating equation (7) where $\bar{\rho}=\bar{\rho}_{i}$ and using equation (15) for $\bar{\rho}_{i}$ :

$$
\begin{aligned}
\dot{\bar{\delta}}_{i}= & \bar{h}_{0} \dot{\bar{h}}_{i}+\left(\bar{\omega}_{1} \bar{\rho}_{p} n \cos n \theta_{i}\right)+\frac{1}{2}\left\{\dot{\bar{x}} \cos \theta_{i}+\dot{\bar{y}} \sin \theta_{i}\right. \\
& \left.+\bar{\omega}_{\mathrm{c}}\left(\bar{y} \cos \theta_{i}-\bar{x} \sin \theta_{i}\right)\right\}
\end{aligned}
$$

where

$$
\dot{\bar{\theta}}_{i}=\bar{\omega}_{\mathrm{c}}, \quad \dot{\bar{\theta}}^{\prime}=\omega_{1}, \quad \theta^{\prime}=\theta_{i}
$$

The second term on the RHS of equation (16) is the rate of change of radial interference $\bar{\rho}_{i}$ where $\bar{\rho}_{p}=0$, because the shaft's wavy contour does not vary with time. This is a reasonable assumption because $n$ is chosen to make the elastostatic contact width much less than the feature wavelength.

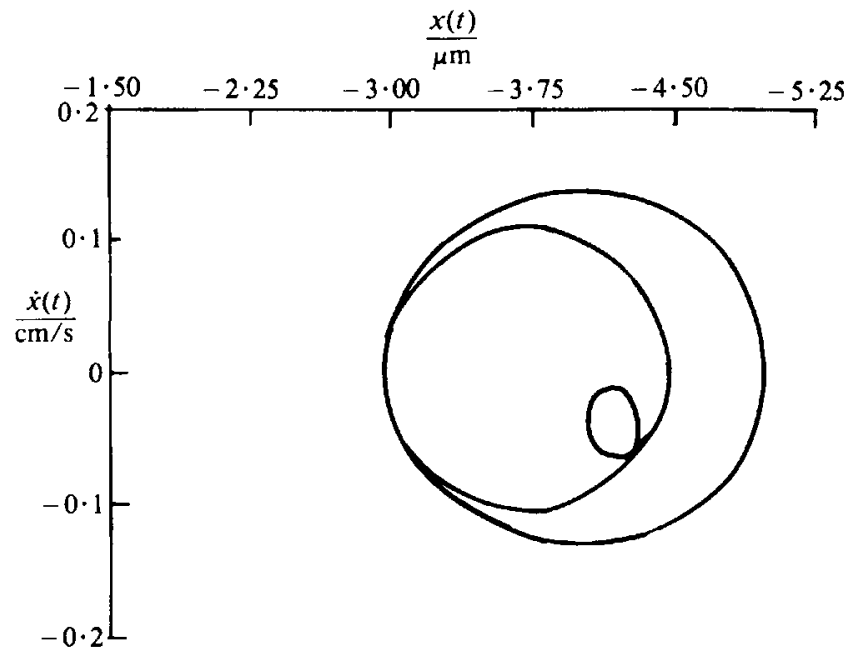

(a) $n=48$

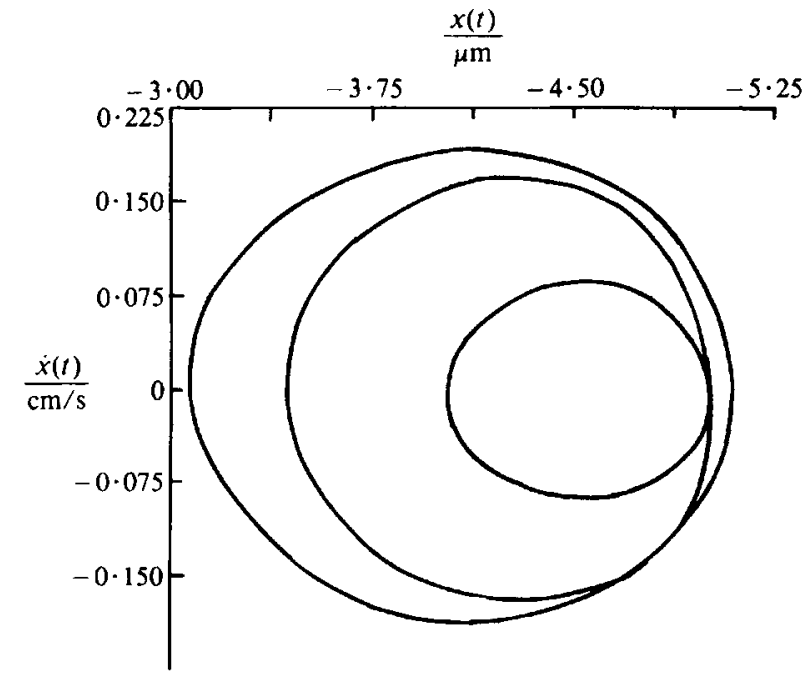

(b) $n=24$

Fig. 14 Steady state phase plane $x$ component trajectory with wavy inner race $\left(Q=500 \mathrm{~N}, \rho=5 \mu \mathrm{m}, \rho_{p}=\right.$ $\sqrt{ } 2 \mu \mathrm{m}, \omega_{1}=209 \mathrm{rad} / \mathrm{s}, m=12$ )
The value of $\rho_{p}$ depends on the accuracy of the machining process. The r.m.s. value of wavy features can be correlated with the shaft diameter (16) when $0.04 \mathrm{~m}<D<0.05 \mathrm{~m}, \rho_{p}$ (r.m.s.) $\simeq 1 \mu \mathrm{m}$. Therefore $\rho_{p} \simeq \sqrt{2 \mu \mathrm{m}}$. Figures $14 \mathrm{a}$ and $14 \mathrm{~b}$ shows the phase plane response for two values of $n$. The smallest loop corresponds to the forcing frequency introduced by the waviness. Here the wave-pass frequency (number of wave peaks passing the eye per second) is $1956 \mathrm{~Hz}$ (calculated from the phase plane it is 1587.5 ). The outer loop represents ball-pass frequency of $159 \mathrm{~Hz}$ and the middle loop is the system base frequency of $260 \mathrm{~Hz}$. The static deflection position is still the same as in Fig. 5, and the forced vibration has not significantly affected the $x$ amplitude because its frequency far exceeds the model base frequency. Halving $n$ has increased the maximum $x$ amplitude and has also altered the relative loop disposition. The $x$ direction vibration amplitude has not become excessive on account of the surface waviness, probably because the oil films and elastic deflection have absorbed some of the input amplitude. The system is analogous to a car with a soft suspension going over a sharply corrugated road.

A fast Fourier transform spectral analysis was then made on the response of the shaft centre to the wavy inner race example above. Figure 15 shows that ballpass frequency is significant. The wave-forcing frequency has little effect, as was shown in Fig. 14a. A sub-harmonic of the ball-pass frequency has also appeared. No response above the forcing frequency is apparent, although in a real bearing this may be the case because of its more complex arrangement.

Experiments on ball bearings subjected to an axial preload and having wavy surface features (1) exhibit a more complex frequency response spectrum, probably because both their inner and outer races are wavy and are not generally in phase. The simple model used here nevertheless indicates some significant frequencies which affect the shaft motion.

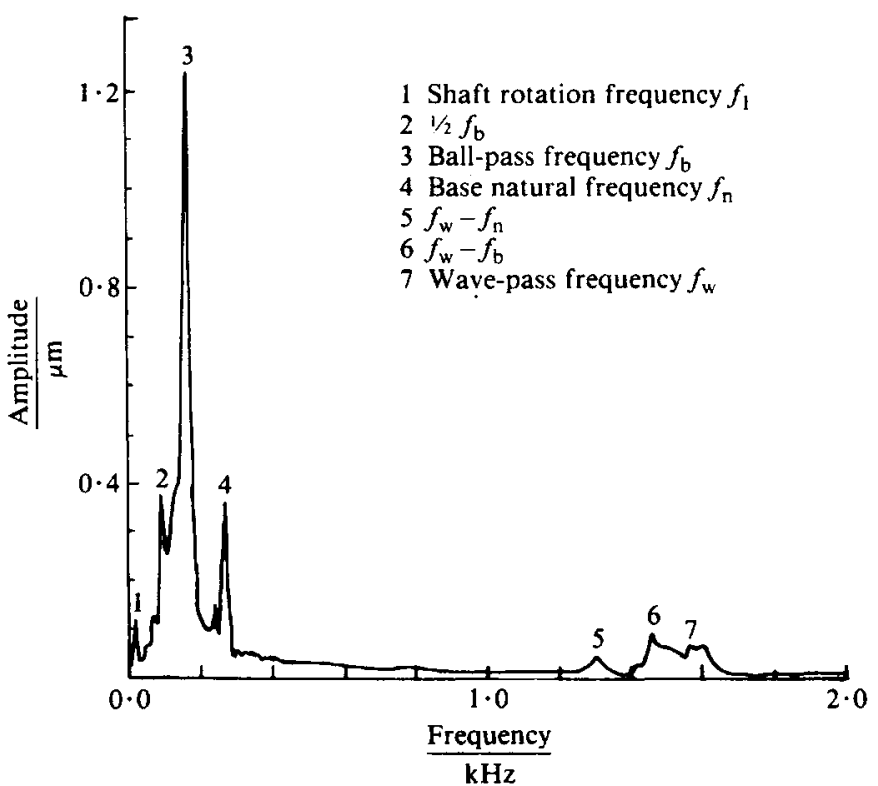

Fig. 15 Steady state frequency spectrum of $x$ oscillations with wavy inner race $\left(Q=500 \mathrm{~N}, \rho=5 \mu \mathrm{m}, \rho_{p}=\right.$ $\sqrt{ } 2 \mu \mathrm{m}, \omega_{1}=209 \mathrm{rad} / \mathrm{s}, m=12, n=48$ ) 


\section{CONCLUSION}

The vibration characteristics of lubricated radial deepgroove ball bearings which support a horizontal rigid rotating shaft have been studied when it is subjected to various external excitations. The variable compliance vibration response under the weight of the shaft itself shows a limit cycle in the steady state operating at a quasi-simple harmonic natural frequency. The limit cycle frequency and amplitude is affected by the number of balls, applied load and radial internal clearance. These in their turn determine the magnitude of the squeeze film damping characteristic between the balls and their races.

When an external periodic forcing function is applied to the shaft by rotating unbalance the ball-pass frequency becomes significant. When the inner race is given a prescribed circumferential surface waviness a response frequency equalling the shaft speed times the number of waves becomes significant in addition to the ball-pass frequency.

\section{REFERENCES}

1 Wardle, F. P. and Poon, S. Y. Rolling bearing noise-cause and cure. Chart. Mech. Engr, July/August 1983.

2 Dareing, D. W. and Johnson, K. L. Fluid film damping of rolling contact vibrations. J. Mech. Engng Sci., 1975, 17 (4).

3 Walford, T. L. H. and Stone, B. J. Measurement of radial stiffness of rolling element bearings under oscillating conditions. J. Mech. Engng Sci., 1980, 22 (4).

4 Walford, T. L. H. and Stone, B. J. The sources of damping in rolling element bearings under oscillating conditions. Proc. Instn. Mech. Engrs, Dec. 1983, 197C.

5 Lacey, S. J., Wardle, F. P. and Poon, S. Y. High speed bearings for C.N.C. machine tool spindles. Chart. Mech. Engr, Dec. 1983.

6 Herrebrugh, K. Elastohydrodynamic squeeze films between two cylinders in normal approach. Trans. ASME, J. Lubric. Tech., April 1970.

7 Christensen, H. E.H.L. theory of spherical bodies in normal approach. Trans. ASME, J. Lubric. Tech., Jan. 1970.

8 Mostofi, A. Oil film thickness and pressure distribution in e.h.l. contacts. PhD thesis, 1981, Imperial College, University of London.

9 Mostofi, A. and Gohar, R. Oil film thickness and pressure distribution elliptical contacts. J. Mech. Engng Sci., 1982, 24(4), 173-182.

10 Ranger, A. P., Ettles, C. M. and Cameron, A. The solution of the point contact E.H.L. problem. Proc. R. Soc. Lond., 1975, A346, 227-244.

11 Harris, T. A. Rolling bearing analysis, 1966, (John Wiley).

12 Timoshenko, S. and Weaver, W. and Young, D. H. Vibration problems in engineering, 1974, 4th edition (John Wiley).

13 Rahnejat, H. Influence of vibration on the oil film in concentrated contacts. PhD thesis, submitted January 1984, Imperial College, London.

14 Buzzachi, G., Cartoceti, M., de Michellis, G. and Sala, C. Infield experience in condition monitoring of rotating machinery by demodulated resonance analysis. J. Acoustic Emission, 1983, 2 $(1 / 2)$.

15 Cole, E. B. Theory of vibrations for engineers, 1935 (Crosby, Lakewood and Son, London).

16 Thomas, T. R. (Ed.) Rough surfaces, 1982 (Longman).

17 Cameron, A. The principles of lubrication, 1966 (Longmans Green).

\section{APPENDIX 1}

The hydrodynamic reaction in equation (3) contains the effect of both entraining and squeezing motions, such that

$$
\bar{W}_{i}=\bar{W}_{\mathrm{si}}+\bar{W}_{\mathrm{R} i}
$$

where $\mathrm{s} \equiv$ squeeze and $\mathrm{R} \equiv$ roll.
From (17), which assumes half Sommerfeld conditions,

$$
W_{\mathrm{R} i}=\frac{6 \sqrt{2 \pi U \eta_{0}}}{(3+2 \bar{R}) h_{i}^{1 / 2}}\left(\frac{R_{x}^{3}}{\bar{R}}\right)^{1 / 2}
$$

where $\bar{R}=R_{x} / R_{y}$ and $h_{i}$ is the minimum film thickness at time $t$. To obtain $W_{\text {si }}$, assume it may be found by considering the squeeze only of a rigid egg-shaped body of principle radii of curvature $R_{x}$ and $R_{y}$ approaching a rigid plane at velocity $w_{s i}=\partial h_{i} / \partial t$, where conditions are isoviscous. The Reynolds equation for pure squeeze under isoviscous conditions is

$$
\frac{\partial}{\partial x}\left(\frac{h^{3}}{\eta_{0}} \frac{\partial \rho}{\partial x}\right)+\frac{\partial}{\partial y}\left(\frac{h^{3}}{\eta_{0}} \frac{\partial p}{\partial y}\right)=12 \frac{\partial h_{i}}{\partial t}
$$

Let

$$
\begin{aligned}
& \bar{x}=\frac{x}{\sqrt{ }\left(2 R_{x} h_{i}\right)}, \quad \bar{y}=\frac{y}{\sqrt{\left(2 R_{y} h_{i}\right)}} \\
& \bar{p}=\frac{p h_{i}^{2}}{12 R_{x} \eta_{0} \partial h / \partial t}, \quad \bar{h}_{i}=\frac{h_{i}}{h_{0}} \quad \text { and } \quad \bar{R}=\frac{R_{x}}{R_{y}}
\end{aligned}
$$

therefore,

$$
\frac{\partial}{\partial \bar{x}}\left(\bar{h}^{3} \frac{\partial \bar{p}}{\partial \bar{x}}\right)+\bar{R}\left(\bar{h}^{3} \frac{\partial \bar{p}}{\partial \bar{y}}\right)=2
$$

where $h=h_{i}+x^{2} / 2 R_{x}+y^{2} / 2 R_{y}$.

Guess the form of $\bar{p}$ by assuming it to be

$$
\bar{p}=\frac{K}{\left(1+\bar{x}^{2}+\bar{y}^{2}\right)^{2}}
$$

Substituting equation (20) into equation (19) yields

$$
K=-\frac{1}{2(1+\bar{R})}
$$

which satisfies the guess. Therefore,

$$
\bar{p}=\frac{1}{2(1+\bar{R})\left(1+\bar{x}^{2}+\bar{y}^{2}\right)^{2}}
$$

The integrated pressure $W_{s i}$ can now be obtained from

$$
W_{\mathrm{si}}=\int_{x=-\infty}^{\infty} \int_{y=-\infty}^{\infty} p \mathrm{~d} x \mathrm{~d} y
$$

or, expressed non-dimensionally,

$$
\frac{W_{\mathrm{s} i} h_{i}(\bar{R})^{1 / 2}}{24 R_{x}^{2} \eta_{0}} \frac{\partial t}{\partial h_{i}}=\left(-\frac{1}{1+\bar{R}}\right) \iint_{-\infty}^{+\infty} \frac{\mathrm{d} \bar{x} \mathrm{~d} \bar{y}}{\left(1+\bar{x}^{2}+\bar{y}^{2}\right)^{2}}
$$

Integrating the RHS first with respect to $\bar{x}$ we get

$$
\mathrm{RHS}=-\frac{\pi}{2(1+\bar{R})} \int_{0}^{\infty} \frac{\mathrm{d} \bar{y}}{\left(1+\bar{y}^{2}\right)^{3}}
$$

Upon substitution, $\bar{y}=\tan \Phi$ and integrating with respect to $\bar{y}$, the RHS comes to RHS $=-\pi /\{2(1+\bar{R})\}$; so equation (22) can be written as

$$
W_{\mathrm{s} i}=-\frac{9 \pi^{2} R_{x}^{2} \eta_{\mathrm{o}} U}{2(1+\bar{R})(\bar{R})^{1 / 2} h_{i}}\left(\frac{\partial h_{i}}{\partial t}\right)
$$


Thus equation (17) becomes, in terms of the nondimensional groups and normalized variables,

$$
\begin{aligned}
\bar{W}_{i}= & \left(\frac{12 \pi}{\bar{R}^{1 / 2} h_{0}^{* 1 / 2}} \frac{U^{*}}{W_{0}^{*}}\right) \\
& \left\{\frac{1}{\sqrt{2(3+2 \bar{R})(\bar{h}})^{1 / 2}}-\frac{3 \pi \omega_{\mathrm{s} i}^{*}}{8 h_{0}^{1 / 2}(1+\bar{R}) \bar{h}_{i}}\right\}
\end{aligned}
$$

where $h_{0}^{*}$ is the initial condition minimum film thickness. Equation (24) is cumbersome for numerical manipulation so let it be replaced by an exponential

$$
\bar{W}_{i}=\frac{C_{\text {iso }}\left(1-B_{0} \mathrm{e}^{\left.\overline{\bar{h}_{i}}\right) 1 / 2}\right.}{\left(\bar{h}_{i}\right)^{1 / 2}}
$$

where

$$
C_{\mathrm{iso}}=\frac{12 \pi U^{*}}{\sqrt{2} \bar{R} h_{0}^{*}(3+2 \bar{R})}
$$

The maximum percentage error between equations (24) and (25) is about 5 per cent for a wide range of values of $w_{\mathrm{s} i}^{*}, U^{*}$ and $\bar{h}_{i}$.

\section{APPENDIX 2}

For large positive values of $\overline{\bar{h}}_{i}, \bar{W}_{i}$ in equation (3) is replaced by an approximate expression

$$
\bar{W}_{i}=\frac{C_{3}}{\overline{\bar{h}}_{i}}+C_{2}
$$

which ensures that $\bar{W}_{i}$ can never become negative.

Let $\bar{h}_{c}$ and $\bar{W}_{\mathrm{c}}$ be respectively the speed and load at which equation (26) starts to operate. So from equations (3) and (26), using the hydrodynamic part of (3),

$$
\psi_{2}\left(\frac{1-B_{0} \mathrm{e}^{\lambda \bar{h}_{\mathrm{c}}}}{\dot{\bar{h}}_{i}}\right)^{1 / 2}=\frac{C_{3}}{\dot{\bar{h}}_{\mathrm{c}}}+C_{2}
$$

Also, the slopes must be the same at the junction. As $\bar{h}_{i}$ is constant along any curve in Fig. 2, at $\bar{h}_{\mathrm{c}}$,

$$
\frac{\partial \bar{W}_{i}}{\partial \bar{h}_{i}}=-\frac{\psi_{2} B_{0} \bar{\lambda} \mathrm{e}^{\bar{\lambda} \bar{h}_{\mathrm{c}}}}{2 \bar{h}_{i}^{1 / 2}}\left(1-B_{0} \mathrm{e}^{\bar{\lambda} \bar{h}_{\mathrm{c}}}\right)^{-1 / 2}
$$

and also at $\bar{h}_{\mathrm{c}}$,

$$
\frac{\partial W_{i}}{\partial \dot{h}_{i}}=-\frac{C_{3}}{\vec{h}_{i}^{2}}
$$

from equation (26). Therefore,

$$
C_{3}=\frac{\psi_{2} B_{0} \bar{\lambda} \bar{h}_{\mathrm{c}}^{2} \mathrm{e}^{\overline{\lambda \bar{h}_{\mathrm{c}}}}}{2 \bar{h}_{i}^{1 / 2}\left(1-B_{0} \mathrm{e}^{\bar{\lambda} \bar{h}_{\mathrm{c}}}\right)^{1 / 2}}
$$

A suitable value for $\dot{\bar{h}}^{\text {c }}$ can be obtained from equation (24) by finding the limiting value of $h_{i}$ to make $\bar{W}_{i}=0$, where

$$
\omega_{s i}^{*}=\frac{\omega_{s i}}{U}=\frac{\tau h_{0} \hat{h}_{i}}{U}
$$

Let the limiting value be $h_{i e}$. Thus:

$$
\dot{h}_{i e}=\frac{8}{3 \pi}\left(\frac{U^{2} M \delta_{0}}{2 R_{x} W_{0} h_{0}}\right)^{1 / 2} \frac{1+2 \bar{R}}{3+2 \bar{R}}\left(\bar{h}_{i}\right)^{1 / 2}
$$

Now let

$$
\dot{\bar{h}}_{\mathrm{c}}=0.999 \dot{\bar{h}}_{i e}
$$

which makes $\bar{W}_{i}$ just positive. Hence $C_{2}$ and $C_{3}$ can be found from equations (27) and (28).

\section{APPENDIX 3}

The governing equations of motion in two degrees of freedom \{i.e. equations (8) and (9)\} are solved by an iterative procedure which incorporates the equations (3) and (7). The step-by-step integration, known as the constant average acceleration method $(12,17)$ uses the principles of the trapezoidal rule. The acceleration in each suitably small time step is taken to be the average of its respective values at the beginning and the end of the same interval. Although this method is unconditionally stable, it is somewhat inaccurate when small variations of small quantities are to be detected. Therefore, we use the linear acceleration method (here referred to as the moving average acceleration) where the acceleration changes linearly within each small time step $\delta \bar{t}$. However, it has been shown in (17) that this method is only conditionally stable, which means that care must be taken in its use.

In the present analysis, values of $\ddot{\bar{x}}_{k, j}$ and $\ddot{\bar{y}}_{k, j}$ at any given time are obtained from equations (8) and (9) respectively. Then, the step-by-step integration follows as below:

For the $\bar{x}$ oscillations $(12,17)$ :

$$
\dot{\bar{x}}_{k, j}=\dot{\bar{x}}_{k-1}+\left(\ddot{\bar{x}}_{k-1}+\ddot{\bar{x}}_{k,(j-1)}\right) \frac{\delta \bar{t}}{2}
$$

and

$$
\bar{x}_{k, j}=\bar{x}_{k-1}+\left(2 \dot{x}_{k-1}+\dot{\bar{x}}_{k, j}\right) \frac{\delta \bar{t}}{3}+\ddot{\bar{x}}_{k-1} \frac{\delta \bar{t}^{2}}{6}
$$

For $k>1$, when $k=1$ :

$$
\dot{\bar{x}}_{1, j}=\dot{\bar{x}}_{0}+\left(\ddot{\bar{x}}_{0}+\ddot{\bar{x}}_{1, j-1}\right) \frac{\delta \bar{t}}{2}
$$

and

$$
\bar{x}_{1, j}=\bar{x}_{0}+\left(2 \dot{\bar{x}}_{0}+\dot{\bar{x}}_{1, j}\right) \frac{\delta \bar{t}}{3}+\ddot{\bar{x}}_{0} \frac{\delta \bar{t}^{2}}{6}
$$

where $\bar{x}_{0}, \dot{\bar{x}}_{0}$ are input conditions and $\ddot{\ddot{x}}_{0}$ is given by equation (8) at $\bar{t}=0$. $\dot{\bar{x}}$ terms are initialized at the beginning of each time step $k$ where $j=1$ as below.

When $k=1$ :

$$
\dot{\bar{x}}_{1,1}=\dot{\bar{x}}_{0}+\ddot{\bar{x}}_{0} \delta \bar{t}
$$

When $k=2$ :

$$
\dot{\bar{x}}_{2,1}=\dot{\bar{x}}_{0}+2 \ddot{\bar{x}}_{0} \delta \bar{t}
$$

and for $k>2$ :

$$
\dot{\bar{x}}_{k, 1}=\dot{\bar{x}}_{k-2}+2 \ddot{\bar{x}}_{k-1} \delta \bar{t}
$$

Similar expressions are used for the $\vec{y}$ oscillations and for the small oil roof ripple oscillations $\bar{S}$ and $\dot{\bar{S}}$, with 
the inclusion of a third index, $i$, identifying a specific ball contact. As an example:

$$
\bar{S}_{i, k, j}=\bar{S}_{i,(k-1)}+\left(2 \dot{\bar{S}}_{i,(k-1)}+\bar{S}_{i, k, j}\right) \frac{\delta \bar{t}}{3}+\dot{S}_{i,(k-1)} \frac{\delta \bar{t}^{2}}{6}
$$

The $\ddot{\bar{S}}_{i, k, j}$ terms are obtained by equating the differentials of equations (3) and (10) with respect to $\bar{t}$. Thus:

$$
\begin{aligned}
& \ddot{\bar{S}}_{i, k, j}=-\frac{\bar{\sigma}_{2 i}+\bar{\sigma}_{3_{i}}}{\bar{\sigma}_{1_{i}}} \\
& \bar{\sigma}_{1_{i}}=\alpha^{\prime} \bar{\beta} \psi_{1} \bar{A}_{i}\left(1-\bar{A}_{i}\right)^{\alpha^{\prime}-1}\left(1-\bar{B}_{i}\right)^{1 / 2} \bar{h}_{i}+\bar{\lambda} \psi_{2} \bar{B}_{i} \bar{h}_{i}^{\alpha^{\prime}+1} \\
& \sigma_{2_{i}}=\bar{h}_{i}^{\alpha_{1}{ }^{\prime}-1}\left(1-\bar{B}_{i}\right)^{1 / 2} \dot{\bar{\delta}}_{i} \bar{\delta}_{i}^{\gamma-1} \\
& \bar{\sigma}_{3_{i}}=\bar{S}_{i}\left(1-\bar{B}_{i}\right)^{1 / 2}\left\{\alpha^{\prime} \psi_{1}\left(1-\bar{A}_{i}\right)^{\alpha^{\prime}} \bar{h}_{i}+\psi_{2} \bar{h}_{i}^{\alpha^{\prime}}\left(1-\bar{B}_{i}\right)\right\}
\end{aligned}
$$

and

$$
\alpha_{1}^{\prime}=\frac{2 \alpha^{\prime}+5}{2}
$$

when

$$
\begin{array}{ll}
\bar{t}=0, & \bar{\sigma}_{30}=0, \quad \bar{A}_{i}=\bar{B}_{i}=0.75 \\
\text { and } & \bar{h}_{i}(0)=1
\end{array}
$$

and when $\dot{S}_{i e}=\dot{h}_{i e}$, the differential of equation (26) replaces that of equation (3). Therefore:

$$
\ddot{\bar{S}}_{i, k, j}=-\frac{\gamma \dot{\bar{\delta}}_{i} \bar{\delta}_{i}^{\gamma}-1 \dot{\bar{h}}_{i}^{2}}{C_{3}}
$$

